\title{
Multiple Visual Rating Scales Based on Structural MRI and a Novel Prediction Model Combining Visual Rating Scales and Age Stratification in the Diagnosis of Alzheimer's Disease in the Chinese Population
}

\author{
Zhenhua Yuan ${ }^{1}$, Chuzheng Pan ${ }^{1}$, Tingting Xiao ${ }^{1}$, Menghui Liu ${ }^{2}$, Weiwei Zhang ${ }^{2}$, \\ Bin Jiao ${ }^{1,3,4}$, Xinxiang Yan ${ }^{1,3,4}$, Beisha Tang ${ }^{1,3,4,5,6,7}$ and Lu Shen ${ }^{1,3,4,8 *}$ \\ ${ }^{1}$ Department of Neurology, Xiangya Hospital, Central South University, Changsha, China, ${ }^{2}$ Department of Radiology, Xiangya \\ Hospital, Central South University, Changsha, China, ${ }^{3}$ National Clinical Research Center for Geriatric Disorders, Central South \\ University, Changsha, China, ${ }^{4}$ Key Laboratory of Hunan Province in Neurodegenerative Disorders, Central South University, \\ Changsha, China, ${ }^{5}$ Parkinson's Disease Center of Beijing Institute for Brain Disorders, Beijing, China, ${ }^{6}$ Collaborative \\ Innovation Center for Brain Science, Shanghai, China, ${ }^{7}$ Collaborative Innovation Center for Genetics and Development, \\ Shanghai, China, ${ }^{8}$ Key Laboratory of Organ Injury, Aging and Regenerative Medicine of Hunan Province, Changsha, China
}

OPEN ACCESS

Edited by:

Rolf Andreas Heckemann, University of Gothenburg, Sweden

Reviewed by:

Jordi A. Matias-Guiu, Hospital Clínico San Carlos, Spain Xiao-Ping Wang,

Shanghai Jiao Tong University, China

*Correspondence: Lu Shen

shenlu@csu.edu.cn

Specialty section: This article was submitted to Applied Neuroimaging, a section of the journal Frontiers in Neurology

Received: 11 August 2018 Accepted: 23 January 2019 Published: 20 February 2019

Citation:

Yuan Z, Pan C, Xiao T, Liu M, Zhang $W$, Jiao $B$, Yan $X$, Tang $B$ and Shen L (2019) Multiple Visual Rating Scales Based on Structural MRI and a Novel Prediction Model Combining

Visual Rating Scales and Age Stratification in the Diagnosis of Alzheimer's Disease in the Chinese

Population. Front. Neurol. 10:93. doi: 10.3389/fneur.2019.00093
Objective: To explore the value of multiple visual rating scales based on structural MRI in the diagnosis of Alzheimer's disease (AD) in the Chinese population.

Materials and Methods: One hundred patients with $A D$ and 100 age- and gender-matched cognitively normal controls were enrolled in this study. All the participants underwent neuropsychological tests and a structural MRI scan of the brain, among them, $42 \mathrm{AD}$ cases and 47 cognitively normal controls also underwent 3D-T1 weighted sequence used for the analysis of voxel-based morphometry (VBM). The $A D$ cases were divided into mild and moderate-severe groups according to the mini-mental state examination. Each participant was evaluated by two trained radiologists who were blind to the clinical information, according to the six visual rating scales, including for medial temporal lobe atrophy (MTA), posterior atrophy (PA), anterior temporal (AT), orbitofrontal (OF) cortex, anterior cingulate (AC), and fronto-insula (FI). Finally, we estimated the relationship between the visual rating scales and the volume of corresponding brain regions, using correlation analysis, and evaluated the specificity and sensitivity of every single scale and combination of multiple scales in the diagnosis of $A D$, using a receiver operating characteristic $(R O C)$ curve and establishing a logistic regression model.

Results: The optimal cutoff of all six visual rating scales for distinguishing AD cases from normal controls was 1.5. Using automated classification based on all six rating scales, the accuracy for distinguishing $A D$ cases from healthy controls ranged from 0.68 to 0.80 (for mild AD) and $0.77-0.90$ (for moderate-severe AD), respectively. A diagnostic prediction model with a combination of MTA and OF results was made as follows: Score $=\mathrm{B}_{\mathrm{MTA}(\text { score) }}+\mathrm{B}_{\mathrm{OF} \text { (score) }}-1.58$ (age $<65$ years); Score $=\mathrm{B}_{\mathrm{MTA} \text { (score) }}+\mathrm{B}_{\mathrm{OF} \text { (score) }}-4.09$ (age $\geq 65$ years). The model was superior to any single visual rating scale in the diagnosis of mild $A D(P<0.05)$. 
Conclusion: Each of the six visual rating scales could be applied to the diagnosis of moderate-severe $A D$ alone in the Chinese population. A prediction model of the combined usage of MTA, OF, and age stratification for the early diagnosis of AD was preliminarily established.

Keywords: visual rating scales, Alzheimer's disease, sensitivity, specificity, Chinese population, prediction model

\section{INTRODUCTION}

Alzheimer's disease is a neurodegenerative disorder mainly characterized by an insidious but progressive loss of memory, accompanied by personality changes and behavior disorders. It is the most common type of dementia in the elderly, and the prevalence is $11 \%$ in those over the age of 65 years, and as high as $32 \%$ in those over the age of 85 years (1). Early diagnosis of $\mathrm{AD}$ is of great importance to the treatment, management, and prognosis $(2,3)$. Molecular biomarkers contributing to the diagnosis of $\mathrm{AD}$ are becoming available but are not widely used in clinical practice. As a common screening means of $\mathrm{AD}$, structural magnetic resonance imaging (MRI) plays a key role in the diagnosis of $\mathrm{AD}$ and has been included in the diagnostic guidelines (4-6). Although a number of sophisticated analysis methods are available to quantify global and regional atrophy from MRI, visual rating scales are highly efficient, rapid, and practical tools in clinical practice.

At the earliest, Scheltens et al. put forward a visual rating scale used to evaluate medial temporal lobe atrophy (MTA) in 1992 (7). The sensitivity and specificity of MTA were 81 and $67 \%$, respectively, so it was considered one of the image markers of AD. In many studies, the rating scale (MTA) was subsequently applied. Recently, it has been included into the diagnostic guidelines for $\operatorname{AD}(4,6,8)$. In 2011, Koedam et al. put forward another evaluation method, posterior atrophy (PA), focusing on the structural changes of the posterior cingulate sulcus, precuneus, parieto-occipital sulcus, and the parietal cortex (9). The sensitivity and specificity were 58 and $95 \%$, respectively. In addition, several other visual rating scales including anterior temporal (AT), orbitofrontal cortex (OF), anterior cingulate (AC) and fronto-insula (FI) were consecutively put forward (1014). Recently, through evaluation of T1-weighted imaging in 184 post-mortem confirmed dementia patients, Harper et al. found that the combination of six visual rating scales was better than any single rating scale in the diagnosis and differential diagnosis of $\mathrm{AD}$ (15). The sensitivity and specificity of the established equation based on six visual rating scales were 94 and 89\%, respectively, in distinguishing $\mathrm{AD}$ patients from normal controls.

However, no study has stated the value of multiple visual rating scales in the diagnosis of $\mathrm{AD}$ in China. To address this gap, we conducted a study to explore the value of multiple visual rating scales based on structural MRI in the diagnosis of $\mathrm{AD}$ in the Chinese population and to combine the aforementioned visual rating scales to establish a simple and effective prediction model for early diagnosis of $\mathrm{AD}$.

\section{MATERIALS AND METHODS}

\section{Subjects}

This study included $102 \mathrm{AD}$ cases (mild AD: 43, moderate and severe AD: 59). All the patients were enrolled in the Department of Neurology of Xiangya Hospital of Central South University, either in the outpatient or inpatient setting between May 2015 and April 2017. The study also included 101 age-matched, gender-matched and cognitively normal controls from the Health Management Center of Xiangya Hospital. This study was approved by the Ethics Committee of Xiangya Hospital of Central South University in China (equivalent to an Institutional Review Board) and was carried out in accordance with the approved guidelines and regulations. Written informed consent was obtained from each participant.

Inclusion criteria of $\mathrm{AD}$ cases:

1) All the patients should meet the diagnostic criteria of "clinical probable AD" established by the National Institute of Neurological and Communicative Disorders and Stroke and the Alzheimer's Disease and Related Disorders Association (NINCDS-ADRDA) in 2007 (4).

2) All the patients underwent the neuropsychological tests, including the Mini-Mental State Examination (MMSE), Activity of Daily Living (ADL) scale, Clinical Dementia Rating (CDR) and Neuropsychiatric Inventory (NPI).

3) Each participant underwent a structural imaging scan of brain through 3.0 Tesla MRI (Signa HDX, General Electric Healthcare, Milwaukee, WI, United States).

\section{Exclusion criteria of AD cases:}

1) Patients with dementia caused by cerebral vascular diseases, poisoning, central nervous system infection, anemia, trauma, and other diseases and patients with other degenerative dementias (frontotemporal dementia, dementia with Lewy body and so on).

2) The patients who could not complete the MRI scan due to embedded metal objects in the body (dentures, stents, pacemaker, metal fixtures).

3) Pregnant and lactating women.

4) Subjects with a severe systemic disease (patients with severe hepatic disease, or a long history of chronic hepatic disease and the alanine aminotransaminase (ALT) and aspartate aminotransaminase (AST) exceed the 1.5 times the upper limit; patients with renal dysfunction; with uncontrolled hypertension; with uncontrolled hyperglycemia; with severe cardiac, pulmonary, or hematological diseases). 
Inclusion criteria for cognitively normal controls:

1) No complaint of cognitive impairment.

2) The score of the MMSE test was in the normal range.

3) Age, sex, and the years of education should be matched with the $\mathrm{AD}$ cases.

Exclusion criteria of cognitively normal controls:

1) Subjects who could not complete the MRI scan due to embedded metal objects in the body (dentures, stents, pacemaker, metal fixtures).

2) Pregnant and lactating women.

3) Subjects with a severe systemic diseases (patients with severe hepatic disease, or a long history of chronic hepatic disease and the alanine aminotransaminase (ALT) and aspartate aminotransaminase (AST) exceed the 1.5 times the upper limit; patients with renal dysfunction; with uncontrolled hypertension; with uncontrolled hyperglycemia; with severe cardiac, pulmonary, or hematological diseases).

\section{MRI Scanning}

Brain MR imaging was performed on a 3.0 T MRI scanner (Signa EXCITE, General Electric, Fairfield, Connecticut, United States) at Xiangya Hospital Imaging Center. For all MRI procedures, the head was immobilized using self-expanding foam cushions. Volumetric (3D) T1-weighted images were acquired: with thickness/gap $=3 / 1 \mathrm{~mm}$, echo time $(\mathrm{TE})=30 \mathrm{~ms}$, pulse repetition time $(\mathrm{TR})=2,000 \mathrm{~ms}$, TI $=380 \mathrm{~ms}$, flip angle $=$ $90^{?}$, matrix $=64 \times 64$, field of view $(\mathrm{FOV})=220 \times 220 \mathrm{~mm}$, voxel size $=0.5 \times 0.5 \times 0.5 \mathrm{~mm}$. All the participants underwent structural MRI brain scanning (including T1 weighted sequence, T2 weighted sequence, T2 FLAIR sequence and T1 weighted coronal thin layer scanning), among them, $42 \mathrm{AD}$ cases and 47 normal controls also underwent 3D-T1 weighted sequence used for the analysis of voxel-based morphometry (VBM).

\section{Data Collection of Visual Rating Scales Evaluation and Voxel-Based Morphometry (VBM)}

First, two radiologists with at least 10 years of working experience in neuroimaging were trained on consistency of visual rating scales evaluation. Subsequently, visual rating of the T1 weighted sequence of all included participants was performed by the two trained radiologists blind to all clinical and pathological information. Six brain regions were rated according to existing scales. Detailed evaluating rules of the six visual rating scales were described in the previous studies $(7,9-14)$ and Figure 1. To improve the consistency of rating, two selected radiologists were trained several times, and slice selection of the structural MRI was specified.

To explore the relationship between each rating scale and pattern of gray matter volume loss, VBM was performed using SPM-8 (Statistical Parametric Mapping, Version8) and MATLAB2010a (uk.mathworks.com/products/matlab). In total, 89 individuals, including $42 \mathrm{AD}$ cases and 47 normal controls, were enrolled to perform the analysis of VBM. Because the preprocessing and analysis of original images varied with different sequences, we needed to classify the original images before processing the data from the MRI. In this study, Dcm2AsiszImg software was used to complete the classification of original images. Before statistical analysis, the classified data should have undergone specified preprocessing achieved by using MATLAB2010a and SPM-8. The processing flow of images included motion correction, spatial normalization and segmentation and smoothness of brain tissue imaging. The realignment of head movement aimed to reduce the impact of noise produced by head movements on signal. We used the EPI template of SPM8 to normalize the image data of all subjects and transformed the original images into template images in units of a volume of $3 \times 3 \times 3 \mathrm{~mm}$. Subsequently, correction for local nonlinear deformation was performed to eliminate local subtle difference and register the data of all subjects into the Montreal Neurological Institute (MNI) space. Gray matter, white matter, and cerebrospinal fluid (CSF) maps were obtained using the unified segmentation approach (16). We used $4 \times 4 \times 4$ full-width half-maximum (FWHM) function to smooth the space to reduce spatial signal-to-noise ratio further and the error caused by space normalization to individuals.

We selected the corresponding brain regions of MTA, PA, OF, AC, AT, and FI, then used Representational State Transfer (REST) software to make a mask. Eventually, the mask was used to extract the gray matter signal of corresponding brain regions. The maps of gray matter signal of corresponding brain regions are presented in Figure 1.

\section{Consistency Evaluation}

\section{The Assessment of Consistency Between Raters}

Intra-class correlation (ICC) is one of the reliability coefficients to evaluate the interobserver reliability and test-retest reliability. The value of ICC ranges from 0 to 1 . A value of ICC lower than 0.4 indicates poor reliability, a range from 0.4 to 0.75 indicates ordinary reliability, and higher than 0.75 indicates good reliability. It is generally acknowledged that the value of ICC should be higher than $0.70(17,18)$.

\section{Correlation Analysis Between the Score of Each Visual Rating Scale and Gray Matter Signal of Corresponding Brain Region}

To ascertain whether the visual rating scales can really reflect the atrophy of corresponding brain regions, we took the intracranial volume, age, and gender as control variables, and performed partial correlation analysis to estimate the correlation between the score of each visual rating scale and the gray matter signal of the corresponding brain region.

\section{Exploration of the Value of a Single Visual Rating Scale in the Diagnosis of AD}

According to the evaluation results of each visual rating scale, the receiver operating characteristic (ROC) curve was drawn to ascertain the optimal cutoff to diagnose $\mathrm{AD}$, and the sensitivity, specificity, and area under the ROC (AUC) of each visual rating scale were calculated respectively. 


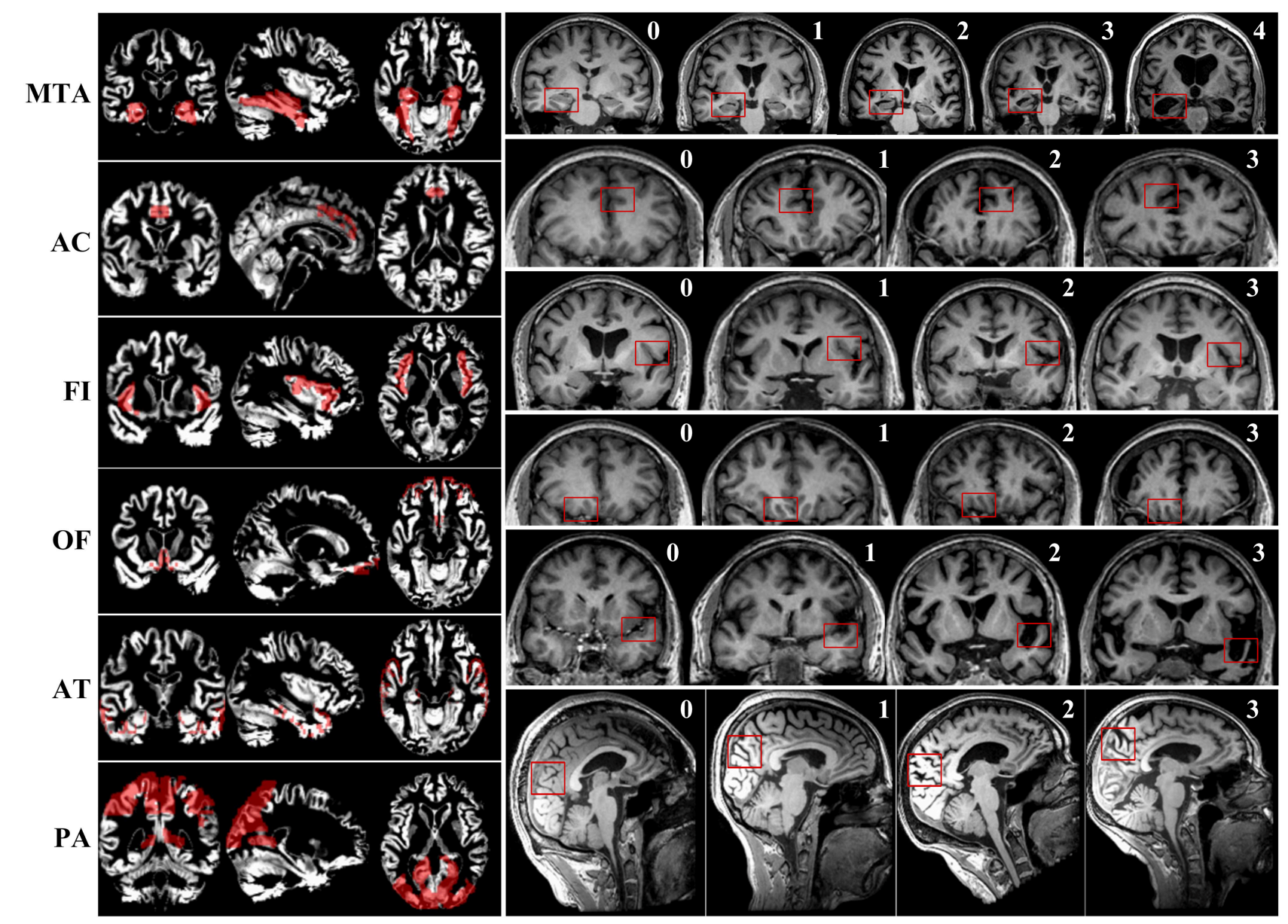

FIGURE 1 | The maps of gray matter signal of corresponding brain regions and examples of scoring of six visual rating scales.

\section{Exploration of the Value of Combining Multiple Visual Rating Scales in the Early Diagnosis of Alzheimer's Disease (Establishment of the Logistic Regression Equation)}

The individuals were divided into two groups according to age (age $\geq 65$ years; age $<65$ years). The scores (rounded to the nearest integer) of the six visual rating scales and age were enrolled as the concomitant variables of the regression equation. Given that the preceding variables were ordered multivariate statistics, each visual rating scale was set as a dummy variate and diagnosis was set as the dependent variate. A model was established through stepwise selection of the binary logistic regressions. Finally, the optimal model that could distinguish the mild $\mathrm{AD}$ cases from the normal controls was ascertained according to the variation of $-2 \log$ likelihood.

\section{Statistical Analysis}

All data processing and analyses were performed using SPSS v.21.0 (IBM, West Grove, Pennsylvania, USA) software. Measurement data were presented as means and standard deviations (SDs), and categorical data were presented as proportions. Differences of the measurement variables were tested using two-sample Student's $t$-test or analysis of variance test. Differences of the categorical variables were tested by the 2 tests. Differences of the ranked data were compared using the Wilcoxon rank sum test. The correlation analysis of the two groups of measurement data was performed using partial correlation analysis. The correlation analysis of categorical variables was performed using logistic regression analysis. For all statistical tests, $p<0.05$ was considered significant.

\section{RESULTS}

\section{Demographic and Clinical Data}

In the early quality control of the imaging data sets, three subjects ( 2 patients and 1 normal control) were excluded due to excessive head movements during the MRI scan. Ultimately, a total of 200 subjects were enrolled in this study, including 100 patients meeting the diagnostic criteria of "clinical probable AD" and 100 age- and gender- matched healthy controls. The demographic and clinical data of the enrolled subjects are described in Table 1. 


\section{Assessment of Consistency Between Raters}

The value of ICC in this study ranged from 0.70 to 0.83 , which indicates a good consistency of rating between raters. The detailed information is shown in Table 2.

\section{Correlation Analysis Between Each Visual Rating Scale and MMSE}

All six visual rating scales have a negative correlation with scores of the MMSE. The correlation coefficient range was $-0.35 \sim$ -0.48 and was statistically significant $(p<0.05)$. The detailed information is shown in Table 3.

\section{VBM and Correlation Analysis Between the Score of Each Visual Rating Scale and the Volume of Gray Matter of Corresponding Brain Regions}

In total, 89 individuals, including $42 \mathrm{AD}$ cases and 47 normal controls, were enrolled to perform the analysis of VBM. The overview of the participants' clinical data is described in Table 4. Research results indicated that there was a significant negative correlation $(p<0.05)$ between each visual rating scale and the volume of gray matter of the corresponding brain regions. Detailed information is described in Table 5.

TABLE 1 | Demographic and clinical data of the AD cases and normal controls.

\begin{tabular}{|c|c|c|c|c|}
\hline & $A D$ cases & Normal controls & $p$-value & Cohen'd \\
\hline Number & 100 & 100 & & \\
\hline Age (years) & $63.44 \pm 9.97$ & $63.46 \pm 8.17$ & $0.99^{a}$ & \\
\hline Gender (M/F) & $31 / 69$ & $35 / 65$ & $0.65^{b}$ & \\
\hline MMSE & $15.70 \pm 6.60$ & $27.50 \pm 1.30$ & - & \\
\hline Education (years) & $9.73 \pm 2.76$ & $9.07 \pm 2.90$ & $0.72^{\mathrm{a}}$ & \\
\hline CDR & $1.47 \pm 0.61$ & - & - & \\
\hline ADL & $28.13 \pm 8.4$ & - & - & \\
\hline NPI & $10.78 \pm 13.1$ & - & - & \\
\hline MTA_L & $2.32 \pm 1.05$ & $1.03 \pm 0.54$ & $<0.01^{\mathrm{C}}$ & 1.545 \\
\hline MTA_R & $2.45 \pm 1.06$ & $1.04 \pm 0.62$ & $<0.01^{\mathrm{c}}$ & 1.624 \\
\hline PA_L & $2.21 \pm 0.81$ & $1.31 \pm 0.63$ & $<0.01^{c}$ & 1.240 \\
\hline PA_R & $2.21 \pm 0.81$ & $1.31 \pm 0.63$ & $<0.01^{c}$ & 1.240 \\
\hline AT_L & $2.01 \pm 0.73$ & $1.13 \pm 0.64$ & $<0.01^{\mathrm{C}}$ & 1.282 \\
\hline AT_R & $2.00 \pm 0.70$ & $1.06 \pm 0.53$ & $<0.01^{c}$ & 1.514 \\
\hline OF_L & $1.96 \pm 0.74$ & $0.90 \pm 0.44$ & $<0.01^{\mathrm{C}}$ & 1.741 \\
\hline OF_R & $1.96 \pm 0.76$ & $0.88 \pm 0.47$ & $<0.01^{\mathrm{C}}$ & 1.709 \\
\hline AC_L & $1.87 \pm 0.77$ & $0.85 \pm 0.48$ & $<0.01^{\mathrm{C}}$ & 1.590 \\
\hline AC_R & $1.90 \pm 0.77$ & $0.84 \pm 0.49$ & $<0.01^{c}$ & 1.642 \\
\hline FI_L & $2.11 \pm 0.77$ & $1.17 \pm 0.57$ & $<0.01^{c}$ & 1.456 \\
\hline Fl_R & $2.11 \pm 0.79$ & $1.16 \pm 0.56$ & $<0.01^{\mathrm{C}}$ & 1.449 \\
\hline
\end{tabular}

${ }^{a} p$-value was calculated by student's t-test.

${ }^{b} p$-value was calculated by $x^{2}$-tests.

${ }^{c} p$-value was calculated by Wilcoxon rank sum test.

M, male; F, female; FL, Left; $R$, Right.

\section{The Value of a Single Visual Rating Scale in the Diagnosis of Mild and \\ Moderate-Severe AD}

The patients with $\mathrm{AD}$ were divided into two groups (mild $\mathrm{AD}$, moderate-severe $\mathrm{AD}$ ) according to the MMSE scores. Mild AD was defined as follows: 18 points $\leq$ MMSE $\leq 23$ points (19). The age- and gender-matched cognitively normal subjects were selected randomly as the controls. The concrete clinical data are described in Tables 6, 7.

\begin{tabular}{lll} 
TABLE 2 | Assessment of consistency of rating between raters. & \\
\hline visual rating scales & position & ICC \\
\hline MTA & Left & 0.83 \\
& Right & 0.82 \\
PA & Left & 0.74 \\
& Right & 0.74 \\
AT & Left & 0.74 \\
& Right & 0.75 \\
OF & Left & 0.70 \\
& Right & 0.70 \\
AC & Left & 0.71 \\
& Right & 0.70 \\
FI & Left & 0.77 \\
& Right & 0.75
\end{tabular}

TABLE 3 | Correlation analysis between each visual rating scale and MMSE.

\begin{tabular}{lcc}
\hline Visual rating scales & Correlation coefficient & $\boldsymbol{p}^{\mathbf{a}}$ \\
\hline MTA & -0.41 & $<0.01$ \\
PA & -0.37 & $<0.01$ \\
AT & -0.42 & $<0.01$ \\
OF & -0.35 & $<0.01$ \\
AC & -0.48 & $<0.01$ \\
FI & -0.40 & $<0.01$
\end{tabular}

${ }^{a}$ After correction of age and gender.

TABLE 4 | Demographic and clinical data of the AD cases and normal controls.

\begin{tabular}{lccc}
\hline & AD cases & Normal controls & $\boldsymbol{p}$-value \\
\hline Number & 42 & 47 & \\
Age & $59.38 \pm 8.70$ & $57.21 \pm 8.37$ & $0.234^{\mathrm{a}}$ \\
Sex(M/F) & $17 / 25$ & $14 / 33$ & $0.374^{\mathrm{b}}$ \\
Education (years) & $9.80 \pm 2.68$ & $9.08 \pm 2.82$ & $0.848^{\mathrm{a}}$ \\
MMSE & $16.87 \pm 6.33$ & $27.22 \pm 1.30$ & - \\
CDR & $1.02 \pm 0.50$ & - & - \\
ADL & $27.80 \pm 7.41$ & - & - \\
NPI & $8.02 \pm 9.60$ & - & - \\
\hline
\end{tabular}

a $p$-value was calculated by student's test.

${ }^{b} p$-value was calculated by $x^{2}$-tests.

M, male; F, female. 
TABLE 5 | Correlation analysis between each visual rating scale and the volume of gray matter of corresponding brain regions.

\begin{tabular}{lcc}
\hline $\begin{array}{l}\text { Corresponding brain region vs. } \\
\text { visual rating scale }\end{array}$ & Correlation coefficient & $\boldsymbol{p}^{\text {-value }} \mathbf{a}^{\mathbf{a}}$ \\
\hline MTA & -0.50 & $<0.01$ \\
PA & -0.45 & $<0.01$ \\
AT & -0.22 & 0.0042 \\
OF & -0.30 & $<0.01$ \\
AC & -0.31 & $<0.01$ \\
FI & -0.42 & $<0.01$ \\
\hline
\end{tabular}

${ }^{a}$ After correction of age, gender and intracranial volume.

TABLE 6 | Clinical data of the mild AD cases and normal controls.

\begin{tabular}{lccc}
\hline & AD Cases & Normal controls & $\boldsymbol{p}$-value \\
\hline Number & 43 & 43 & \\
Age (years) & $63.48 \pm 10.93$ & $63.00 \pm 9.80$ & $0.819^{\mathrm{a}}$ \\
Sex(M/F) & $11 / 32$ & $14 / 29$ & $0.635^{\mathrm{b}}$ \\
MMSE & $21.12 \pm 2.78$ & $27.52 \pm 1.30$ & - \\
CDR & $0.75 \pm 0.35$ & - & - \\
ADL & $22.56 \pm 2.93$ & - & - \\
NPI & $7.88 \pm 9.50$ & - & - \\
\hline
\end{tabular}

${ }^{a} p$-value was calculated by student's test.

${ }^{b} p$-value was calculated by $x^{2}$-tests.

$M$, male; $F$, female.

TABLE 7 | Clinical data of the moderate and severe AD cases and normal controls.

\begin{tabular}{lccc}
\hline & AD cases & Normal controls & $\boldsymbol{p}$-value \\
\hline Number & 57 & 57 & \\
Age (years) & $63.80 \pm 7.20$ & $63.42 \pm 9.29$ & $0.80^{\mathrm{a}}$ \\
Sex (M/F) & $20 / 37$ & $21 / 36$ & $1.00^{\mathrm{b}}$ \\
MMSE & $11.60 \pm 4.32$ & $27.47 \pm 1.32$ & - \\
CDR & $2.00 \pm 0.60$ & - & - \\
ADL & $32.24 \pm 8.81$ & - & - \\
NPI & $12.96 \pm 14.51$ & - & - \\
\hline
\end{tabular}

${ }^{a} p$-value was calculated by student's test.

${ }^{b} p$-value was calculated by $\chi^{2}$-tests.

$M$, male; $F$, female.

The optimal cutoff of all six visual rating scales which could distinguish moderate-severe $\mathrm{AD}$ cases from normal controls was 1.5. The sensitivity, specificity, and AUC of all six visual rating scale ranges were $0.51-0.72,0.56-0.97$, and $0.68-0.80$, respectively. Among them, the AUC of MTA and OF ranked the highest and were both 0.80 . The detailed data are described in Table 8.

The optimal cutoff of all six visual rating scales, which could distinguish moderate-severe $\mathrm{AD}$ cases from normal controls was 1.5. The sensitivity, specificity, and AUC of all the six visual rating scale ranges were $0.78-0.87,0.68-0.95$, and $0.77-0.90$, respectively. Among them, the AUC of MTA, AC, and OF ranked
TABLE 8 | The AUC, sensitivity and specificity of single visual rating scale in the mild AD cases.

\begin{tabular}{|c|c|c|c|c|c|}
\hline Visual rating scale & position & Cutoff & sensitivity & specificity & AUC \\
\hline \multirow[t]{3}{*}{ MTA } & $\mathrm{L}$ & 1.5 & 0.63 & 0.95 & 0.79 \\
\hline & $\mathrm{R}$ & 1.5 & 0.67 & 0.90 & 0.80 \\
\hline & M & 1.5 & 0.62 & 0.95 & 0.79 \\
\hline \multirow[t]{3}{*}{ PA } & $\mathrm{L}$ & 1.5 & 0.72 & 0.56 & 0.69 \\
\hline & $\mathrm{R}$ & 1.5 & 0.72 & 0.56 & 0.69 \\
\hline & M & 1.5 & 0.72 & 0.56 & 0.69 \\
\hline \multirow[t]{3}{*}{ AT } & $L$ & 1.5 & 0.65 & 0.66 & 0.68 \\
\hline & $\mathrm{R}$ & 1.5 & 0.67 & 0.86 & 0.77 \\
\hline & M & 1.5 & 0.65 & 0.86 & 0.77 \\
\hline \multirow[t]{3}{*}{ OF } & $\mathrm{L}$ & 1.5 & 0.65 & 0.97 & 0.80 \\
\hline & $\mathrm{R}$ & 1.5 & 0.62 & 0.97 & 0.79 \\
\hline & $M$ & 1.5 & 0.63 & 0.96 & 0.79 \\
\hline \multirow[t]{3}{*}{$A C$} & $L$ & 1.5 & 0.51 & 0.95 & 0.75 \\
\hline & $\mathrm{R}$ & 1.5 & 0.51 & 0.95 & 0.76 \\
\hline & $\mathrm{M}$ & 1.5 & 0.51 & 0.95 & 0.76 \\
\hline \multirow[t]{3}{*}{$\mathrm{FI}$} & $L$ & 1.5 & 0.62 & 0.73 & 0.70 \\
\hline & $\mathrm{R}$ & 1.5 & 0.62 & 0.73 & 0.70 \\
\hline & $M$ & 1.5 & 0.60 & 0.75 & 0.70 \\
\hline
\end{tabular}

$L$, left; $R$, right; $M$, mean.

the highest and were all 0.90 . The detailed data are described in Table 9.

\section{The Value of a Prediction Model Combining Multiple Visual Rating Scales in the Diagnosis of Mild AD}

Through the analysis of binary logistic regressions, three concomitant variables were enrolled, including two visual rating scales (MTA, OF) and age. The diagnostic prediction model was established as follows: Score $=\mathrm{B}_{\mathrm{MTA} \text { (score) }}+\mathrm{B}_{\mathrm{OF} \text { (score) }}-1.58$ (age $<65$ years); Score $=\mathrm{B}_{\mathrm{MTA} \text { (score) }}+\mathrm{B}_{\mathrm{OF} \text { (score) }}-4.09$ (age $\geq 65$ years). When the value of the model $\geq 0$, the person is estimated to have $\mathrm{AD}$, when the value of the model $<0$, the person is estimated to be cognitively normal. The concrete parameters are described in detail in Tables 10, 11. The sensitivity, specificity, and AUC of this model in distinguishing mild AD cases from normal controls were $0.74,0.93$, and 0.92 , respectively. Compared to the most effective single visual rating scale, MTA (AUC: 0.79, sensitivity: 0.62, specificity: 0.95), the difference between them was statistically significant $(p<0.05)$.

\section{DISCUSSION}

As one of the common screening means, structural MRI of the brain plays a key role in the diagnosis and differential diagnosis of dementia (20-22). Visual rating scales based on structural MRI could increase the accuracy of imaging assessment and provide radiologists and other clinical researchers a framework to describe the structural image.

Evaluation of the visual rating scales was inevitably subjective; however, two studies $(23,24)$ indicated that two trained 
TABLE 9 | The AUC, sensitivity and specificity of single visual rating scale in the moderate and severe AD cases.

\begin{tabular}{|c|c|c|c|c|c|}
\hline Visual rating scale & Position & Cutoff & Sensitivity & Specificity & AUC \\
\hline \multirow[t]{3}{*}{ MTA } & $L$ & 1.5 & 0.86 & 0.86 & 0.89 \\
\hline & $\mathrm{R}$ & 1.5 & 0.87 & 0.85 & 0.90 \\
\hline & $\mathrm{M}$ & 1.5 & 0.86 & 0.89 & 0.90 \\
\hline \multirow[t]{3}{*}{ PA } & $\mathrm{L}$ & 1.5 & 0.86 & 0.74 & 0.85 \\
\hline & $\mathrm{R}$ & 1.5 & 0.86 & 0.74 & 0.85 \\
\hline & M & 1.5 & 0.86 & 0.74 & 0.85 \\
\hline \multirow[t]{3}{*}{ AT } & $L$ & 1.5 & 0.84 & 0.80 & 0.86 \\
\hline & $\mathrm{R}$ & 1.5 & 0.82 & 0.85 & 0.86 \\
\hline & $\mathrm{M}$ & 1.5 & 0.82 & 0.91 & 0.89 \\
\hline \multirow[t]{3}{*}{ OF } & $L$ & 1.5 & 0.82 & 0.95 & 0.90 \\
\hline & $\mathrm{R}$ & 1.5 & 0.80 & 0.93 & 0.89 \\
\hline & M & 1.5 & 0.82 & 0.94 & 0.90 \\
\hline \multirow[t]{3}{*}{$A C$} & $L$ & 1.5 & 0.79 & 0.95 & 0.90 \\
\hline & $\mathrm{R}$ & 1.5 & 0.78 & 0.94 & 0.90 \\
\hline & $\mathrm{M}$ & 1.5 & 0.80 & 0.95 & 0.90 \\
\hline \multirow[t]{3}{*}{$\mathrm{FI}$} & $L$ & 1.5 & 0.84 & 0.68 & 0.77 \\
\hline & $\mathrm{R}$ & 1.5 & 0.83 & 0.68 & 0.77 \\
\hline & $\mathrm{M}$ & 1.5 & 0.84 & 0.84 & 0.88 \\
\hline
\end{tabular}

$L$, left; $R$, right; $M$, mean.

TABLE 10 | Setting list of dummy variable parameters.

\begin{tabular}{|c|c|c|c|c|c|}
\hline & & \multicolumn{4}{|c|}{ Dummy variable parameters } \\
\hline & & $(1)$ & (2) & (3) & (4) \\
\hline \multirow[t]{5}{*}{ MTA } & 0 & 0 & 0 & 0 & 0 \\
\hline & 1 & 1 & 0 & 0 & 0 \\
\hline & 2 & 0 & 1 & 0 & 0 \\
\hline & 3 & 0 & 0 & 1 & 0 \\
\hline & 4 & 0 & 0 & 0 & 1 \\
\hline \multirow[t]{4}{*}{ OF } & 0 & 0 & 0 & 0 & \\
\hline & 1 & 1 & 0 & 0 & \\
\hline & 2 & 0 & 1 & 0 & \\
\hline & 3 & 0 & 0 & 1 & \\
\hline \multirow[t]{2}{*}{ Age } & $\geq 65$ & 1 & & & \\
\hline & $<65$ & 0 & & & \\
\hline
\end{tabular}

radiologists could have good consistency. Our study showed similar results. Consequently, we can conclude that the visual rating scales have a good repeatability and can be applied to clinical practice.

One of the most widely used screening tools for $\mathrm{AD}$, the MMSE, was established by Folstein in 1975 (25). The MMSE covers multiple cognitive domains, including orientation, memory, attention and calculation power, executive functioning, language and visuospatial functioning. The results of our study showed that all six visual rating scales had a negative correlation with the MMSE score. Our findings align with the fact that the MMSE is a comprehensive rating scale covering multiple cognitive domains and the atrophy of corresponding brain regions can decrease the points of corresponding cognitive
TABLE 11 | Variables and related coefficient of regression equation.

\begin{tabular}{lll}
\hline & B & OR \\
\hline MTA(0) & & \\
MTA(1) & 0.78 & 2.2 \\
MTA(2) & 4.31 & 74.56 \\
MTA(3) & 21.08 & $3.50 \times 10^{9}$ \\
MTA(4) & 20.06 & $5.13 \times 10^{8}$ \\
OF(0) & & \\
OF(1) & 0.17 & 1.19 \\
OF(2) & 1.78 & 5.94 \\
OF(3) & 22.02 & $3.65 \times 10^{9}$ \\
Age ( $\geq 65$ years) & -2.51 & 0.82 \\
Constant & -1.58 & \\
\hline B. & &
\end{tabular}

$B$, regression coefficient; OR, odds ratio.

domains. A previous study indicated that MTA and PA were negatively correlated with MMSE scores independently in AD cases (9), which was consistent with our study.

The visual rating scale of MTA was first put forward by Scheltens et al., and the sensitivity and specificity of MTA in distinguishing $\mathrm{AD}$ from normal controls were 81 and $67 \%$, respectively (7). In our study, the sensitivity and specificity of MTA were $(62 \%, 95 \%)$ in mild AD cases and $(86 \%, 89 \%)$ in moderate and severe $\mathrm{AD}$ cases. The subtle difference between the two studies may be due to the stratification of $\mathrm{AD}$ according to severity of disease. The previous study indicated that the sensitivity and specificity of FI in distinguishing early-onset AD from normal controls were 74 and 94\%, respectively (15), close to our results (84 and $84 \%$ ). AT was mainly used to estimate the atrophy of the temporal lobe in frontotemporal dementia (FTD) cases. A previous study indicated that the scores of AT related to the extent of atrophy at autopsy (11). However, AT could effectively differentiate AD from normal controls in our study, indicating that atrophy of the anterior temporal lobe can be found in $\mathrm{AD}$ cases. Anterior cingulate plays a key role in execution function. One study indicated that the volume of the anterior cingulate cortex had a negative correlation with two items of execution function in amnestic mild cognitive impairment (aMCI) cases, and it decreased significantly compared with normal controls (26). The significant difference of $\mathrm{AC}$ between $\mathrm{AD}$ cases and normal controls in our study was similar to the results of the preceding study. The visual rating scale, PA, focusing on the posterior cingulate, precuneus, parietooccipital sulcus, and parietal cortex, was put forward by Koedam et al. (9). To date, as the only visual rating scale targeting the posterior portion of the brain, PA can be used to differentiate $\mathrm{AD}$ from normal controls and other dementias. The sensitivity and specificity of PA in distinguishing AD from normal controls were 58 and $95 \%$, respectively (9), which was superior to the performance in mild $\mathrm{AD}$ cases and inferior to the performance in moderate and severe AD cases in our study. Atrophy of multiple brain regions, including the insula lobe, anterior hippocampus, temporal pole, and orbital-frontal cortex, was found in AD cases compared to normal controls (12). The discovery indicates that 
there may be diffuse atrophy in $\mathrm{AD}$ brains, which is consistent with the high sensitivity and specificity of each single visual rating scale in distinguishing moderate and severe $\mathrm{AD}$ from normal controls in our study.

In our study, six visual rating scales and age were enrolled in the logistic regression equation to explore a prediction model that could distinguish mild $\mathrm{AD}$ cases from normal controls. The ultimate prediction model included three variables: MTA, $\mathrm{OF}$, and age stratification. The sensitivity and specificity of this model in distinguishing mild $\mathrm{AD}$ cases from normal controls were 0.74 and 0.93 , respectively, superior to the most effective single visual rating scale, MTA (sensitivity: 0.62, specificity: 0.95), and the difference between them was statistically significant $(p$ $<0.05$ ). To date, many studies confirm that medial temporal atrophy is related to $\operatorname{AD}(27,28)$. MTA was verified as practical and repeatable in clinical practice, and was related to the volume of the hippocampus $(29,30)$. As well, increasing evidence indicated that the orbital frontal cortex was involved in the early stage of $\mathrm{AD}(31,32)$. These studies presented corresponding morphological changes of the medial temporal lobe and orbital frontal lobe cortex in the early stage of $\mathrm{AD}$. Therefore, the combined use of MTA and OF may be superior to a single visual rating scale in the early diagnosis of $\mathrm{AD}$ and the higher the score of the model, the greater the possibility of AD.

Age is one of the influencing factors in brain atrophy, and visual rating scales after age stratification would have better accuracy $(15,33,34)$. A previous study indicated that the score of MTA in the normal controls under the age of 70 years ranged from 0 to 1 , and the score of MTA in the normal controls at the age of 70-80 years ranged from 0 to 2 (35). Given that there was research taking 65 years old as the cutoff point in the process of combining six visual rating scales to diagnose AD (15), our study divided $\mathrm{AD}$ cases into two groups (early-onset $\mathrm{AD}$ and late-onset $\mathrm{AD}$ ) and enrolled age as a variate into the research model to be selected. The results showed that age stratification was enrolled into the prediction model finally, and the regression coefficient of age stratification was negative, which indicated that brain atrophy to some extent in the aged cases may be normal senile atrophy.

Our study found that every single visual rating scale could effectively distinguish $\mathrm{AD}$ cases from normal controls and was repeatable in the Chinese population, especially for

\section{REFERENCES}

1. Alzheimer's disease facts and figures. Alzheimers Dement (2016) 12:459-509. doi: 10.1016/j.jalz.2016.03.001

2. de Vries K, Brooker DJ, Smith P. Dementia skills and competencies for primary care liaison: a model for improving identification and timely diagnosis. Prim Health Care Res Dev. (2013) 14:240-9. doi: $10.1017 /$ S1463423612000266

3. Mitchell AJ, Meader N, Pentzek M. Clinical recognition of dementia and cognitive impairment in primary care: a meta-analysis of physician accuracy. Acta Psychiatr Scand. (2011) 124:165-83. doi: 10.1111/j.1600-0447.2011.01730.x

4. Dubois B, Feldman HH, Jacova C, Dekosky ST, Barberger-Gateau P, Cummings J, et al. Research criteria for the diagnosis of Alzheimer's disease: moderate-severe $\mathrm{AD}$ cases. For mild $\mathrm{AD}$ cases, the prediction model of combining MTA, OF, and age stratification was better than using a single visual rating scale. There are still some limitations in our study as follows: The sample size was relatively small and the enrolled cases were mainly clinically probable typical $\mathrm{AD}$ cases. Consequently, a large sample size, detailed age stratification, and enrolling more AD cases, supported by positron emission tomography-computed tomography (PETCT), CSF biomarkers, and autopsy, are necessary to obtain more accurate discoveries.

\section{ETHICS STATEMENT}

All procedures performed in studies involving human participants were approved by the Ethics Committee of Xiangya Hospital, Central South University in China, which was in accordance with ethical standards of the institutional and/or national research committee and with the 1964 Helsinki declaration and its later amendments or comparable ethical standards. Written informed consent was obtained from all subjects.

\section{AUTHOR CONTRIBUTIONS}

LS and TX involved in the study design. ZY and CP were responsible for the enrollment of the participants. $\mathrm{ZY}, \mathrm{CP}, \mathrm{ML}$, and $\mathrm{WZ}$ were responsible for the estimation of the visual rating scales. LS, BT, and XY were responsible for the confirmation of the participants. ZY wrote the manuscript. LS and BJ modified and revised the manuscript. All authors have read and approved the final version of the manuscript.

\section{FUNDING}

This study was supported by the National Natural Science Foundation of China (No. 81471295 and No. 81671075 to LS) and the National Key Plan for Scientific Research and Development of China (No. 2016YFC1306000 to BT).

\section{ACKNOWLEDGMENTS}

We are grateful to all subjects for participation in our study. revising the NINCDS-ADRDA criteria. Lancet Neurol. (2007) 6:734-746. doi: 10.1016/S1474-4422(07)70178-3

5. Hort J, O’Brien JT, Gainotti G, Pirttila T, Popescu BO, Rektorova I, et al. EFNS guidelines for the diagnosis and management of Alzheimer's disease. Eur J Neurol. (2010) 17:1236-48. doi: 10.1111/j.1468-1331.2010.0 3040.x

6. Dubois B, Feldman HH, Jacova C, Hampel H, Molinuevo JL, Blennow $\mathrm{K}$, et al. Advancing research diagnostic criteria for Alzheimer's disease: the IWG-2 criteria. Lancet Neurol. (2014) 13:614-29. doi: 10.1016/S1474-4422(14)70090-0

7. Scheltens P, Leys D, Barkhof F, Huglo D, Weinstein HC, Vermersch P, et al. Atrophy of medial temporal lobes on MRI in "probable" Alzheimer's disease and normal ageing: diagnostic value and neuropsychological correlates. J Neurol Neurosurg Psychiatry (1992) 55:967-972. 
8. Jack CR Jr, Albert MS, Knopman DS, McKhann GM, Sperling RA, Carrillo MC, et al. Introduction to the recommendations from the National Institute on Aging-Alzheimer's Association workgroups on diagnostic guidelines for Alzheimer's disease. Alzheimers Dement (2011) 7:257-62. doi: 10.1016/j.jalz.2011.03.004

9. Koedam EL, Lehmann M, van der Flier WM, Scheltens P, Pijnenburg YA, Fox $\mathrm{N}$, et al. Visual assessment of posterior atrophy development of a MRI rating scale. Eur Radiol. (2011) 21:2618-25. doi: 10.1007/s00330-011-2205-4

10. Davies RR, Kipps CM, Mitchell J, Kril JJ, Halliday GM, Hodges JR. Progression in frontotemporal dementia: identifying a benign behavioral variant by magnetic resonance imaging. Arch Neurol. (2006) 63:1627-31. doi: 10.1001/archneur.63.11.1627

11. Kipps CM, Davies RR, Mitchell J, Kril JJ, Halliday GM, Hodges JR. Clinical significance of lobar atrophy in frontotemporal dementia: application of an MRI visual rating scale. Dement Geriatr Cogn Disord (2007) 23:334-42. doi: $10.1159 / 000100973$

12. Davies RR, Scahill VL, Graham A, Williams GB, Graham KS, Hodges JR. Development of an MRI rating scale for multiple brain regions: comparison with volumetrics and with voxel-based morphometry. Neuroradiology (2009) 51:491-503. doi: 10.1007/s00234-009-0521-z

13. Hornberger M, Savage S, Hsieh S, Mioshi E, Piguet O, Hodges JR. Orbitofrontal dysfunction discriminates behavioral variant frontotemporal dementia from Alzheimer's disease. Dement Geriatr Cogn Disord (2010) 30:547-552. doi: 10.1159/000321670

14. Ambikairajah A, Devenney E, Flanagan E, Yew B, Mioshi E, Kiernan MC, et al. A visual MRI atrophy rating scale for the amyotrophic lateral sclerosis-frontotemporal dementia continuum. Amyotroph Lateral Scler Frontotemporal Degener. (2014) 15:226-34. doi: 10.3109/21678421.2014.880180

15. Harper L, Fumagalli GG, Barkhof F, Scheltens P, O’Brien JT, Bouwman F, et al. MRI visual rating scales in the diagnosis of dementia: evaluation in 184 post-mortem confirmed cases. Brain (2016) 139:1211-25. doi: 10.1093/brain/aww005

16. Ashburner J, Friston KJ. Unified segmentation. Neuroimage (2005) 26:839-51. doi: 10.1016/j.neuroimage.2005.02.018

17. Bartko JJ. The intraclass correlation coefficient as a measure of reliability. Psychol Rep. (1966) 19:3-11. doi: 10.2466/pr0.1966.19.1.3

18. Health measurement scales: a practical guide to their development and use (5th edition). Aust N Z J Public Health (2016) 40:294-95. doi: 10.1111/1753-6405.12484

19. Guo QH, Hong Z, Shi WX, Lu JC, Zhao QH, Lu CZ. Distinguishing the clinical and psychological characteristics between patients with amnestic mild cognitive impairment and very mild Alzheimer disease. Zhongguo Linchuang Kangfu (2006) 10:4-6. doi: 10.3321/j.issn:1673-8225.2006.26.002

20. Rascovsky K, Hodges JR, Knopman, Mendez MF, Kramer JH, Neuhaus J, et al. Sensitivity of revised diagnostic criteria for the behavioural variant of frontotemporal dementia. Brain (2011) 134:2456-2477. doi: 10.1093/brain/awr179

21. McKhann GM, Knopman DS, Chertkow H, Hyman BT, Jack CR Jr, Kawas CH, et al. The diagnosis of dementia due to Alzheimer's disease: recommendations from the National Institute on Aging-Alzheimer's Association workgroups on diagnostic guidelines for Alzheimer's disease. Alzheimers Dement (2011) 7:263-9. doi: 10.1016/j.jalz.2011.03.005

22. Wardlaw JM, Smith EE, Biessels GJ, Cordonnier C, Fazekas F, Frayne R, et al. Neuroimaging standards for research into small vessel disease and its contribution to ageing and neurodegeneration. Lancet Neurol. (2013) 12:822-838. doi: 10.1016/S1474-4422(13)70124-8
23. Harper L, Barkhof F, Fox NC, Schott JM. Using visual rating to diagnose dementia: a critical evaluation of MRI atrophy scales. J Neurol Neurosurg Psychiatry (2015) 86:1225-33. doi: 10.1136/jnnp-2014-310090

24. Lehmann M, Koedam EL, Barnes J, Bartlett JW, Ryan NS, Pijnenburg YA, et al. Posterior cerebral atrophy in the absence of medial temporal lobe atrophy in pathologically-confirmed Alzheimer's disease. Neurobiol Aging (2012) 33:627.e621-627.e612. doi: 10.1016/j.neurobiolaging.2011.04.003

25. Pangman VC, Sloan J, Guse L. An examination of psychometric properties of the mini-mental state examination and the standardized mini-mental state examination: implications for clinical practice. Appl Nurs Res. (2000) 13:209-13. doi: 10.1053/apnr.2000.9231

26. Borsa VM, Della Rosa PA, Catricala E, Canini M, Iadanza A, Falini A, et al. Interference and conflict monitoring in individuals with amnestic mild cognitive impairment: a structural study of the anterior cingulate cortex. J Neuropsychol. (2018) 12:23-40. doi: 10.1111/jnp.12105

27. Erkinjuntti T, Lee DH, Gao F, Steenhuis R, Eliasziw M, Fry R, et al. Temporal lobe atrophy on magnetic resonance imaging in the diagnosis of early Alzheimer's disease. Arch Neurol. (1993) 50:305-10.

28. Kesslak JP, Nalcioglu O, Cotman CW. Quantification of magnetic resonance scans for hippocampal and parahippocampal atrophy in Alzheimer's disease. Neurology (1991) 41:51-4.

29. Bresciani L, Rossi R, Testa C, Geroldi C, Galluzzi S, Laakso MP, et al. Visual assessment of medial temporal atrophy on MR films in Alzheimer's disease: comparison with volumetry. Aging Clin Exp Res. (2005) 17:8-13.

30. Scheltens P, Launer LJ, Barkhof F, Weinstein HC, van Gool WA. Visual assessment of medial temporal lobe atrophy on magnetic resonance imaging: interobserver reliability. J Neurol. (1995) 242:557-560.

31. Grothe M, Zaborszky L, Atienza M, Gil-Neciga E, Rodriguez-Romero R, Teipel SJ, et al. Reduction of basal forebrain cholinergic system parallels cognitive impairment in patients at high risk of developing Alzheimer's disease. Cereb Cortex (2010) 20:1685-95. doi: 10.1093/cercor/bhp232

32. Van Hoesen GW, Parvizi J, Chu CC. Orbitofrontal cortex pathology in Alzheimer's disease. Cereb Cortex (2000) 10:243-251.

33. Van de Pol LA, Hensel A, Barkhof F, Gertz HJ, Scheltens P, van der Flier WM. Hippocampal atrophy in Alzheimer disease: age matters. Neurology (2006) 66:236-8. doi: 10.1212/01.wnl.0000194240.47892.4d

34. Rhodius-Meester HFM, Benedictus MR, Wattjes MP, Barkhof F, Scheltens $\mathrm{P}$, Muller $\mathrm{M}$, et al. MRI visual ratings of brain atrophy and white matter hyperintensities across the spectrum of cognitive decline are differently affected by age and diagnosis. Front Aging Neurosci. (2017) 9:117. doi: 10.3389/fnagi.2017.00117

35. Pereira JB, Cavallin L, Spulber G, Aguilar C, Mecocci P, Vellas B, et al. Influence of age, disease onset and ApoE4 on visual medial temporal lobe atrophy cut-offs. J Intern Med. (2014) 275:317-330. doi: 10.1111/joim. 12148

Conflict of Interest Statement: The authors declare that the research was conducted in the absence of any commercial or financial relationships that could be construed as a potential conflict of interest.

Copyright (C) 2019 Yuan, Pan, Xiao, Liu, Zhang, Jiao, Yan, Tang and Shen. This is an open-access article distributed under the terms of the Creative Commons Attribution License (CC BY). The use, distribution or reproduction in other forums is permitted, provided the original author(s) and the copyright owner(s) are credited and that the original publication in this journal is cited, in accordance with accepted academic practice. No use, distribution or reproduction is permitted which does not comply with these terms. 